\title{
Gaia and the Astrometric Global Iterative Solution
}

\author{
D. Hobbs ${ }^{1}$, L. Lindegren ${ }^{1}$, B. Holl ${ }^{1}$, \\ U. Lammers ${ }^{2}$ and W. O'Mullane ${ }^{2}$ \\ ${ }^{1}$ Lund Observatory, Lund, Sweden \\ email: david@astro.lu.se, lennart@astro.lu.se, berry@astro.lu.se \\ ${ }^{2}$ European Space Astronomy Center, ESA, Spain \\ email: Uwe.Lammers@esa.int, william.omullane@esa.int
}

\begin{abstract}
Gaia is an ESA space astrometry mission due for launch in 2011-12. We describe part of the work carried out in the Gaia Data Processing and Analysis Consortium, namely the Astrometric Global Iterative Solution (AGIS) currently being implemented at the European Space Astronomy Center (ESAC) in Spain and largely based on algorithms developed at Lund Observatory. Some provisional results based on simulated observations of one million stars are presented, demonstrating convergence at microarcsec level independent of initial conditions.
\end{abstract}

Keywords. astrometry, methods: data analysis, methods: numerical, reference systems

\section{The challenge}

The immense volume of data created by Gaia and especially their complex relationships make the data processing requirements amongst the most challenging even by the standards of computational power in the next decade. The raw science data amount to some 100 Terabytes and the total data archive may surpass one Petabyte. The required numerical processing is of the order of $10^{21}$ floating-point operations.

To meet this challenge, the Gaia Data Processing and Analysis Consortium (DPAC) was formed in 2006 (Mignard et al. 2008). A central part of the processing is to determine the accurate spacecraft attitude, geometric instrument calibration and astrometric reference data for a well-behaved subset of all the objects. This core astrometric processing falls within the responsibility of coordination unit CU3 of the DPAC, and is currently being implemented and will be run at the European Space Astronomy Centre (ESAC) near Madrid in Spain. Lund Observatory and ESAC are jointly responsible for providing the framework and algorithms for the implementation of AGIS. Working versions of all the main algorithms are already in place but require further refinement.

ESAC is one of the main data processing centres for DPAC. The current system, a cluster of 18 dual-3.6 GHz Xeon processors, gives an overall performance of 140 Gflops. Many AGIS test runs have already been made on this system, using simulated data for $10^{6}$ objects. Up until Gaia's launch (end of 2011) the system will be scaled and exercised with progressively more complex and realistic simulations of up to $10^{8}$ objects.

\section{The Astrometric Global Iterative Solution (AGIS)}

The core astrometric processing will determine the positions, parallaxes and proper motions for a subset of 'primary sources' (bona-fide single stars and quasars), together with the spacecraft attitude as function of time, and a large number of instrument calibration parameters. These data must be estimated to microarcsecond accuracy, essentially 
by a least-squares adjustment to the measured image positions on the CCDs. The total number of unknowns is of the order of $10^{9}$. Although the resulting system of equations is very sparse, a direct solution taking fully into account the dependencies between all the unknowns seems to be impractical by many orders of magnitude. Consequently, iterative solution methods are used. The currently adopted 'Astrometric Global Iterative Solution' (AGIS) consists of four blocks executed cyclically until convergence is achieved:

- Source Update - determination of the five astrometric parameters $\left(\alpha, \delta, \pi, \mu_{\alpha *}, \mu_{\delta}\right)$ of each primary source

- Attitude Update - determination of the celestial orientation of the instrument axis as a function of time (using spline representations of the attitude quaternion)

- Calibration Update - determination of the geometric calibration parameters (basic angle, CCD geometry, etc.)

- Global Update - determination of a relatively small number of model parameters that are constant throughout the entire mission (e.g., the PPN parameter $\gamma$ ).

In each block, updates are computed based on the results from the other blocks in this and the previous iteration. The system is data driven: each observation is read from disk precisely once per iteration and updated values passed to the other processes sequentially.

The positions and apparent proper motions of quasars are used to connect the solution to the extragalactic reference frame and to determine the (galactocentric) acceleration of the solar system in the cosmological frame from the secularly changing aberration effect.

AGIS will be run in six-month cycles over the Gaia time-line, using progressively larger sets of observational data. Other processes (e.g., photometry) are run in parallel, on synchronised cycles, and their results fed to the astrometric processing of the next cycle (and vice versa) via a common database. The final iterations therefore incorporate data from the whole mission as well as updated information from all the parallel processes.

\section{Some preliminary results}

A number of AGIS tests have been executed on simulated data for $10^{6}$ primary sources and a mission length of 5 years. This typically involved $5 \times 10^{6}$ astrometric parameters (unknowns), $2 \times 10^{7}$ attitude parameters, and some $2 \times 10^{5}$ geometric calibration parameters. All the parameters were given initial (random and/or systematic) errors up to 0.1 arcsec, which were brought down to levels compatible with the expected overall mission accuracy in some 40-50 AGIS iterations. The iterations were interrupted when the mean update in parallax was below $1 \mu$ as. Remaining errors in the astrometric parameters do however show patterns at the level of 10-20 $\mu$ as, which continue to shrink with further iterations. This behaviour is characteristic for the simple iterative scheme used by AGIS, which leads to the errors (and updates) eventually decreasing exponentially by a factor equal to the largest eigenvalue of the iteration matrix. The relatively significant systematic errors still observed after $\sim 50$ iterations reflect the eigenvector associated with the largest eigenvalue, and disappear with further iterations. This property will be used to accelerate the convergence rate of AGIS and to derive alternative iteration methods.

\section{Acknowledgements}

This work is strongly dependent on parallel developments in other coordination units within DPAC, in particular CU1 (System architecture) and CU2 (Data simulations).

\section{References}

Mignard, F., et al. 2008, this volume p.224 